\title{
Epidemiology, diagnosis and management of superficial mycoses: ours three years retrospective analysis
}

\author{
Barbara Pieretti, Marco Moretti, Maria Rita Sadori, Daniela Sbrozzi, Annamaria Masucci, \\ Lucia Finaurini, Simonetta Gasperoni \\ Laboratorio di Patologia Clinica Ospedale "S. Croce"AORMN - Fano (PU)
}

Key words: Superficial mycoses, Dermatomycosis, Epidemiology, Diagnosis

Epidemiologia e diagnostica delle micosi superficiali: analisi retrospettiva

\section{SUMMARY}

Mycotic infections of the skin, hair and nails are disorder with high prevalence. Dermatophytes are the most frequently etiologic agents followed by yeasts and non-dermatophyte moulds. The geographic distribution is variable because of migration, lifestyle and socio-economics conditions.

In this study, based on ours three years retrospective data analysis, we evaluate the epidemiology of etiologic agents, diagnosis and management of superficial mycoses.

Our data and our experience suggest the importance of this microbiological diagnosis and the role of the Clinical Microbiologist to assess with Dermatologist and Primary Care Phyisician an appropriate diagnostic and therapeutic plan.

\section{INTRODUZIONE}

Le patologie correlate alle infezioni fungine della cute e degli annessi cutanei sono frequenti ed ubiquitarie interessando il $20-25 \%$ della popolazione mondiale $(5,10)$ con incidenza in continuo incremento (1).

I principali agenti eziologici sono i dermatofiti antropofili e zoofili appartenenti ai generi Trichophyton, Microsporum ed Epidermophyton $(5,11,17)$, meno frequentemente i miceti filamentosi jalini, dematiacei e lieviti $(11,13,14)$.

L'infezione, limitata allo strato corneo dell'epidermide, alle unghie, ai peli ed ai capelli, è fortemente dipendente dall'interazione tra l'agente eziologico e la suscettibilità dell'ospite ed è notevolmente influenzata dalle condizioni climatico ambientali ed igienico sanitarie $(5,16)$.

I dati epidemiologici evidenziano un'evoluzione spiccata dell'eziologia nelle diverse aree geografiche legata a viaggi in zone endemiche, fenomeni migratori, adozioni internazionali ed in generale ad un aumento degli spostamenti delle popolazioni $(1,5,8,10,15)$. Lo studio si propone di analizzare retrospettivamente la nostra esperienza relativa all'ultimo triennio focalizzando l'attenzione sulle modalità del percorso diagnostico e sull'epidemiologia degli isolati.

\section{MATERIALI E METODI}

Nel periodo luglio 2009 - luglio 2012 sono stati effettuati presso gli ambulatori del laboratorio 808 prelievi per sospetta micosi cutanea o degli annessi su richiesta dermatologica specialistica $(80 \%)$ o del Medico di Medicina Generale (20\%).

Per oltre il $95 \%$ dei pazienti il prelievo del campione e la raccolta delle informazioni clinico-anamnestiche, mediante un'apposita scheda, sono stati eseguiti dal microbiologo clinico. In una contenuta percentuale di casi $(<5 \%)$ il campione è stato consegnato direttamente dal paziente (materiale raccolto dal richiedente o dal paziente con conseguente limitata disponibilità di informazioni).

Degli 808 campioni analizzati 540 (66.8\%) appartengono a soggetti di sesso femminile e 268 $(33.2 \%)$ di sesso maschile. L'età media dei pazienti è 46.8 anni (min $1, \max 91)$.

L'analisi microbiologica è stata condotta mediante microscopia diretta dopo trattamento con $\mathrm{KOH}$ e semina su Sabouraud Destrosio Agar addizionato di cloramfenicolo-gentamicina e Dermatophyte Agar contenente rosso fenolo come indicatore di $\mathrm{pH}$ (bioMérieux) per 21 giorni a $25^{\circ} \mathrm{C}$. La crescita è stata monitorata quotidianamente. La valutazione delle caratteristiche macroscopiche delle colonie cresciute e l'analisi microscopica dopo colorazione con blu di lattofenolo ci hanno permesso di fare diagnosi eziologica.

Sono stati considerati negativi i campioni privi di crescita in entrambi i terreni trascorsi i 21 giorni di incubazione.

\section{Corresponding author: Pieretti Barbara}

Laboratorio Patologia Clinica Ospedale "S.Croce" Azienda Ospedaliera Ospedali Riuniti Marche Nord

Via Vittorio Veneto 2 - 61032 Fano (PU) - Tel.: 072I 882379 - Fax:072I 882304

E-mail: barbara.pieretti@libero.it 


\section{RISULTATI}

Complessivamente sono risultati positivi 384 (47.5\%) campioni. La Tabella 1 riporta gli agenti etiologici isolati con le relative percentuali. Nell' $82 \%$ dei campioni positivi l'infezione è sostenuta da un singolo agente (Aspergillus spp, Epidermophyton floccosum, Microsporum spp, Trichophyton spp, Candida spp, Penicillum spp, Scopularopsis brevicaulis), mentre nel rimanente $18 \%$ sono state rilevate delle coinfezioni tra specie dello stesso genere (Aspergillus spp., dermatofiti) o tra generi diversi (Aspergillus spp e dermatofiti, Candida spp e Aspergillus spp, Candida spp e dermatofiti, altre specie e dermatofiti).

L'identificazione di specie è stata condotta facendo riferimento ai criteri morfologici macro e microscopici delle colonie, alle informazioni cliniche, alla sede d'infezione, all'origine geografica del paziente, e dove possibile alle modalità di parassitismo ectothrix o endothtrix $(3,6,7)$.

In particolare per le specie del genere Aspergillus sono state prese in considerazione le caratteristiche macroscopiche delle colonie valutandone il colore della parte aerea, il grado di crescita (tempo e dimensioni), i margini, la tessitura di superficie e l'aspetto microscopico considerando la struttura delle ife (sottili, settate, con ramificazione dicotomica) e della testa conidiale (conidioforo, vescicola).

Per i dermatofiti la distinzione tra generi è stata fatta in base all'aspetto macroscopico delle colonie (colore, tempi di crescita, consistenza, tessitura) e alle caratteristiche microscopiche di macroconidi e microconidi.

Per i lieviti si sono valutate le caratteristiche morfologico-tintoriali, la capacità di produrre ife o pseudoife e le caratteristiche biochimiche (es.: identificazione con strumentazione automatizzata Vitek).

Gli stessi criteri di valutazione morfologica delle colonie e analisi microscopica sono stati applicati ai funghi non appartenenti ai generi sopraindicati, facendo riferimento a standard nazionali ed internazionali.

È stato inoltre possibile risalire all'origine geografica dei pazienti con campioni positivi rispetto alla popolazione studiata. La distribuzione in base all'origine geografica ha visto una netta prevalenza di italiani (77.4\%) e con percentuali pari all' $8.6 \%, 7.8 \%$ e $6.2 \%$ rispettivamente, pazienti provenienti dall'Est Europea, Africa del Nord e Africa Sub-Sahariana.

\section{DISCUSSIONE}

Le richieste per la ricerca di infezioni di cute ed annessi sono, anche nella nostra realtà, in continuo aumento essendo raddoppiate rispetto al triennio precedente. Il quesito diagnostico posto sia dallo specialista dermatologo che da parte del medico di medicina generale presenta un'accettabile livello di appropriatezza (47.5\% di campioni positivi) considerando che spesso la patologia cutanea e degli annessi implica importanti difficoltà per la diagnosi differenziale.

L'epidemiologia degli isolati è in linea con i dati presenti in letteratura $(5,17)$ ed evidenzia il ruolo eziologico predominante dei dermatofiti $(56.8 \%)$ tra cui i più frequenti sono il Trichophyton rubrum $(19.8 \%)$ e il Trichopython mentagrophytes $(18.2 \%)$ con incidenza singola delle altre specie inferiore al $5 \%$.

Ai dermatofiti seguono rispettivamente in frequenza di isolamento Candida spp (22.4\%) e gli aspergilli (1.0\%). Si sottolinea come nel 18\% dei casi siano state riscontrate coinfezioni principalmente costituite da associazioni lieviti-dermatofiti $(10.4 \%)$ e fra dermatofiti $(5.2 \%)$. Nella definizione e nell'applicazione del percorso diagnostico (dalla raccolta del campione e delle informazioni al referto fino alla consulenza post analitica) il microbiologo clinico gioca un ruolo fondamentale. La raccolta del campione nella quasi totalità dei casi è stata condotta in un ambulatorio dedicato presso il laboratorio.

Questo garantisce l'idoneità quali-quantitativa del campione oltre alla raccolta mediante un'apposita scheda delle informazioni cliniche (anamnesi, sintomatologia, patologie concomitanti, terapie in atto e pregresse). Il quesito diagnostico se non accompagnato dall'ispezione della lesione e dalla raccolta delle informazioni rischia di non essere soddisfatto correttamente.

Spesso infatti si pone la necessità di posticipare la raccolta del campione o ripeterla a distanza da eventuali interventi terapeutici o di toilette della lesione oppure affiancare all'esame micologico quello batteriologico.

Per la fase analitica la letteratura attribuisce una sensibilità del $50-60 \%$ (4) e un valore predittivo negativo dell' $80 \%(2,9)$ alla lettura diretta microscopica dopo trattamento con $\mathrm{KOH}$ e sensibilità del $20-70 \%(4,12)$ con valore predittivo negativo pari al $70 \%(2,9)$ alla metodica colturale. Combinando le due metodologie si ottengono livelli soddisfacenti di sensibilità e specificità.

La completa disponibilità di informazioni consente inoltre di attribuire all'isolato un ruolo di patogeno, contaminante o di flora residente.

La valutazione quotidiana della crescita permette di accorciare i tempi diagnostici con la possibilità di comunicare rapidamente al paziente o al curante gli esiti garantendo, anche per una diagnostica intrinsecamente lenta, la possibilità di intervenire con terapie appropriate nel minor tempo possibile. 
La diagnostica delle infezioni micotiche della cute e degli annessi è a nostro avviso uno dei campi in cui il microbiologo clinico può esercitare le pro- prie competenze concordando e definendo con i clinici di riferimento un appropriato percorso diagnostico e terapeutico.

Tabella I. Distribuzione degli isolati.

\begin{tabular}{|c|c|c|c|c|c|}
\hline & & \# & $\%$ & \# & $\%$ \\
\hline aspergilli & $\begin{array}{l}\text { Aspergillus flavus } \\
\text { Aspergillus fumigatus } \\
\text { Aspergillus niger }\end{array}$ & $\begin{array}{l}1 \\
1 \\
2 \\
\end{array}$ & $\begin{array}{l}0.3 \\
0.3 \\
0.5 \\
\end{array}$ & 4 & 1.0 \\
\hline più aspergilli & A.niger + A.fumigatus & 1 & 0.3 & 1 & 0.3 \\
\hline aspergilli + dermatofiti & $\begin{array}{l}\text { A.flavus }+ \text { T.schoenleinii } \\
\text { A.niger }+ \text { T.mentagrophytes } \\
\text { A.niger }+ \text { T.mentagrophytes } \\
\text { A.niger }+ \text { T.rubrum }\end{array}$ & $\begin{array}{l}1 \\
1 \\
1 \\
1\end{array}$ & $\begin{array}{l}0.3 \\
0.3 \\
0.3 \\
0.3\end{array}$ & 4 & 1.0 \\
\hline dermatofiti & $\begin{array}{l}\text { Epidermophyton floccosum } \\
\text { Microsporum canis } \\
\text { Microsporum audouinii } \\
\text { Microsporum gypseum } \\
\text { Trichophyton mentagrophytes } \\
\text { Trichophyton rubrum } \\
\text { Trichophyton schoenleinii } \\
\text { Trichophyton tonsurans } \\
\text { Trichophyton verrucosum } \\
\text { Trichophyton violaceum }\end{array}$ & $\begin{array}{c}16 \\
18 \\
2 \\
5 \\
70 \\
76 \\
6 \\
12 \\
4 \\
9\end{array}$ & $\begin{array}{c}4.2 \\
4.7 \\
0.5 \\
1.3 \\
18.2 \\
19.8 \\
1.6 \\
3.1 \\
1.0 \\
2.3\end{array}$ & 218 & 56.8 \\
\hline piu' dermatofiti & $\begin{array}{l}\text { E.floccosum }+ \text { M.gypseum } \\
\text { E.floccosum }+ \text { T.mentagrophytes } \\
\text { E.floccosum }+ \text { T.rubrum } \\
\text { E.floccosum }+ \text { T.rubrum }+ \text { T.schoenleinii } \\
\text { E.floccosum }+ \text { T.schoenleinii } \\
\text { T.mentagrophytes }+ \text { T.schoenleinii } \\
\text { T. rubrum }+ \text { T.mentagrophytes } \\
\text { T.rubrum }+ \text { T.schoenleinii } \\
\text { T.rubrum }+ \text { T.tonsurans } \\
\text { T.rubrum }+ \text { T.verrucosum }\end{array}$ & $\begin{array}{l}2 \\
2 \\
4 \\
1 \\
1 \\
2 \\
4 \\
1 \\
1 \\
2\end{array}$ & $\begin{array}{l}0.5 \\
0.5 \\
1.0 \\
0.3 \\
0.3 \\
0.5 \\
1.0 \\
0.3 \\
0.3 \\
0.5\end{array}$ & 20 & 5.2 \\
\hline lieviti & Candida spp & 86 & 22.4 & 86 & 22.4 \\
\hline lieviti + aspergilli & $\begin{array}{l}\text { Candida } \mathrm{spp}+\text { A.niger } \\
\text { Candida } \mathrm{spp}+\text { A.niger/A.fumigatus }\end{array}$ & $\begin{array}{l}1 \\
1 \\
\end{array}$ & $\begin{array}{l}0.3 \\
0.3 \\
\end{array}$ & 2 & 0.5 \\
\hline lieviti + dermatofiti & $\begin{array}{l}\text { Candida } \mathrm{spp}+\text { E. floccosum } \\
\text { Candida } \mathrm{spp}+\text { M.audouinii } \\
\text { Candida } \mathrm{spp}+T . \text { mentagrophytes } \\
\text { Candida } \mathrm{spp}+\text { T.rubrum } \\
\text { Candida } \mathrm{spp}+\text { T.schoenleinii } \\
\text { Candida } \mathrm{spp}+\text { T.tonsurans } \\
\text { Candida } \mathrm{spp}+T . \text { violaceum } \\
\text { Candida } \mathrm{spp}+T . \text { violaceum }+ \text { E.floccosum }\end{array}$ & $\begin{array}{c}2 \\
1 \\
6 \\
20 \\
3 \\
1 \\
6 \\
1 \\
\end{array}$ & $\begin{array}{l}0.5 \\
0.3 \\
1.6 \\
5.2 \\
0.8 \\
0.3 \\
1.6 \\
0.3 \\
\end{array}$ & 40 & 10.4 \\
\hline altro & $\begin{array}{l}\text { Penicillum spp } \\
\text { Scopulariopsis brevicaulis }\end{array}$ & $\begin{array}{l}2 \\
3\end{array}$ & $\begin{array}{l}0.5 \\
0.8\end{array}$ & 5 & 1.3 \\
\hline altro + dermatofiti & $\begin{array}{l}\text { T.mentagrophytes }+ \text { S.brevicaulis } \\
\text { T.schoenleinii }+ \text { S.brevicaulis } \\
\text { T.rubrum }+ \text { T.tonsurans }+ \text { T.violaceum }+ \\
\text { T.mentagrophytes }+ \text { Penicillum spp }\end{array}$ & $\begin{array}{l}2 \\
1 \\
1\end{array}$ & $\begin{array}{l}0.5 \\
0.3 \\
0.3\end{array}$ & 4 & 1.0 \\
\hline
\end{tabular}




\section{BIBLIOGRAFIA}

1. Ameen M. Epidemiology of superficial fungal infections. Clin Dermatol 2010; 28 (4): 197-201.

2. Andrews MD, Burns M. Common Tinea Infection in Children. Am Fam Physician 2008; 15 (10); 1415-20.

3. Andreoni S, Farina C, Lombardi P. Atlante di Micologia Medica. Systems Communicazioni 2003, 240 pagg.

4. Hainer BL. Dermatophyte Infection. Am Fam Physician 2003; 67 (1): 101-9.

5. Havlickova B, Czaika VA, Friedrich M. Epidemiological trends in skin mucose worldwide. Mycoses 2008; 51 (suppl 4): 2-15.

6. http://microbiology.mtsinai.on.ca/mig/index.shtml (ultimo accesso 12 febbraio 2013).

7. http://www.mycology.adelaide.edu.au/ (ultimo accesso 12 febbraio 2013).

8. Juncosa T, Aguilera P, Jean A, Vicente A, Aguilar AC, Fumadó V. Trichophyton violaceum: an emerging patogen. Enferm Infecc Microbiol Clin 2008; 26 (8): 502-4.

9. Karimzadegan-Nia M, Mir-Amin-Mohammadi A, Bouzari N, Firooz A. Comparison of direct smear, culture and histology for the diagnosis of onychomycosis. Australas J Dermatol 2007; 48 (1): 18-21.

10. Kaur R, Kashyap B, Bhalla B. Onychomycois -
Epidemiology, diagnosis and management. Indian Journal of Medical Microbiology 2008; 26 (2): 10816.

11. Kayman T, Sarigüzel FM, Koç AN, Tekinpen FK. Etiological agents of superficial mycoses in Kayseri, Turkey. J Eur Acad Dermatol Venereol 2012; doi: 10.1111/j.14683083.2012.04589.x. [Epub ahead of print].

12. Laury MA, Haneke E, Stobeck K, Martin S, Zimmer B, Romano PS. Methods for diagnosing onychomycosis: a comparative study and review of the literature. Arch Dermatol 2000; 136: 1112-6.

13. López-Jodra O, Torres-Rodríguez JM. Unusual fungal species causing onychomycosis. Rev Iberoam Micol 1999; 16 (S): S11-5.

14. Moreno G, Arenas R. Other fungi causing onychomycosis. Clin Dermatol 2010; 28(2): 160-3.

15. Neupane S, Pokhrel DB, Pokhrel BM. Onychomycosis: a clinic-epidemiological study. Nepal Med Coll J 2009; 11(2): 92-5.

16. SalekArdakani S, Cota E, Bignell E. Host-fungal interactions: key players of antifungal immunity. Expert Rev Anti Infect Ther 2012; 10 (2): 149-51.

17. Simonnet C, Berger F, Gantier JC. Epidemiology of superficial fungal diseases in French Guiana: a three-year retrospective analysis. Med Mycol 2011; 49 (6): 608-11. 